\title{
New Fuzzy Similarity Measure for Restricted Mobile Networks
}

\author{
Wencan Chen ${ }^{1}$, Xiaodong Liu $^{2, \text { a }}$ \\ ${ }^{1}$ College of Information Science and Engineering, Ocean University of China, Qingdao 266100, \\ P.R.China \\ ${ }^{2}$ College of Mathematical Sciences, Ocean University of China, Qingdao 266100, P.R.China \\ aliuxiaodong.ouc@hotmail.com
}

Keywords: Fuzzy set; Restricted Mobile Network; Distance; Similarity.

\begin{abstract}
Similarity-based search has been a hot research topic for a long history, which is widely used in many applications. The large scale Restricted Floating Sensor (RFS) network is an important mod-el in offshore data collection [1]. Due to the mobility and the large number of sensors, improved techniques are needed to deal with uncertainty and mass queries. As a theoretical basis, this paper constructs a new fuzzy similarity measure based on distance. With examples we illustrate that many common similarity functions can be constructed from these measures. From [2] we know our work over distance and similarity is a reasonable generalization and extension of other Fuzzy Sets. This work provides a theoretical guidance for constructing a fuzzy query processing strategy for our RFS networks.
\end{abstract}

\section{Introduction}

There are significant interests in analyzing the siltation of the estuary and harbor. For example, H. H. Harbor, the second largest harbor for coal transportation (6.7 million tons per year) in China, currently suffers from the increasingly severe pr-oblem of silt deposition along its sea route (19 nautical miles long). The sea route has always been threatened by the silt from the shallow sea area. Monitoring sea depth costs this harbor more than 18 million US dollars per year [1]. In 2007, Yunhao Liu proposed a Restricted Floating Sensor (RFS) model [1]. By locating such sensors, the sea depth can be estimated efficiently without the help of extra ranging devices.

Similarity-based technologies are important in RFS networks: firstly, RFSs are deployed on the sea collecting data of silt. The event detection can be considered as the pattern search over time series data, which highly demands fast retrieval to keep the safety of sea route. Thus the retrieval of similar time series is important to RFSs [1]; secondly, RFS suffers long propagation delay and high-energy consumption. There need a scheme can effectively exploit the sharing among queries to greatly reduce the communication cost. Thus similarity-aware query techniques are important to RFSs [3].

Distinguished from traditional stat-ionary or mobile sensor networks, nodes in the RFS network will generally not be stationary at their original places, but float in a restricted area on the sea [1].. This quality poses some unique challenges. For example, let $v_{i j}$ denotes RFS of the network, where $\mathrm{i}$ stand for the layer, and $\mathrm{j}$ for the sequence number. Let $V=\left\{v_{i j}\right\}$. The topology is shown in Fig. 3. Let $S\left(v_{i_{0} j_{0}}\right)=\left\{v_{i j} \mid v_{i j} \in V, i=i_{0}+1, j \in J_{i_{0} j_{0}}\right\}$ denote the aggregation of all child nodes of $v_{i_{0} j_{0}}$ in the RFS network, whe-re $J_{i_{0} j_{0}}$ contains all the sequence number of these nodes. Because of the movement of RFSs, the relation between $S\left(v_{i_{0} j_{0}}\right)$ and $v_{i_{0} j}\left(j \in J_{i_{0} j_{0}}\right)$ is variable. For example $S\left(v_{12}\right)$ can equal to $\left\{v_{22}, v_{23}, v_{24}\right\}$ or $\left\{v_{23}, v_{24}\right\}$ and $v_{31}$ belongs to $S\left(v_{21}\right)$ or $S\left(v_{22}\right)$. As we know fuzzy theory is efficient in treating imprecision. So in this paper we constru-ct a new similarity measure based on distance on VIFS.

Our work is a reasonable extension of those on Fuzzy Set (FS), Intuitionistic Fuzzy Set (IFS) and Interval-Valued Intuitionistic Fuzzy Set (IVIFS). Furthermore it is guidable on constructing similarity-based technologies for RFS networks. 


\section{New Similarity Measure Based on Distance}

In this section, we will propose a general measure of similarity between VIFSs. Firstly we will propose a general distance functions on VIFS.

Theorem 3.1. Let

1. $f\left(x_{1}, x_{2}, \ldots, x_{s}\right):[0,1]^{s} \rightarrow[0,1]$ with properties:

1) $x_{1}=x_{2}=\ldots=x_{s}=0 \Leftrightarrow f\left(x_{1}, x_{2}, \ldots, x_{s}\right)=0$

2) to any $x_{i}(i=1,2, \ldots, s), f$ is a increasing function.

2. to any $d_{i}(A, B)(i=1,2, \ldots, s)$ is a weak distance function and there exist $j \in\{1,2, \ldots, s\}$ satisfies $d_{j}(A, B)$ is a distance function.

Then to any $(A, B) \in \operatorname{VIFS}(X)$,

$$
d(A, B)=f\left(d_{1}(A, B), d_{2}(A, B), \ldots, d_{s}(A, B)\right)
$$

is a distance function.

Prove: for $d_{i}(A, B)$ are $s$ weak distance functions, from theorem 3.1 we get $d(A, B)$ is also a weak distance function. Then we only prove $d(A, B)$ is a distance function, that is to prove

$d(A, B)=0 \Rightarrow A=B$.

As we know

$d(A, B)=0 \Leftrightarrow f\left(d_{1}(A, B), d_{2}(A, B), \ldots, d_{s}(A, B)\right)=0 \quad$ From

$x_{1}=x_{2}=\ldots=x_{s}=0 \Leftrightarrow f\left(x_{1}, x_{2}, \ldots, x_{s}\right)=0$

we get $d_{i}(A, B)=0(i=1,2, \ldots, s)$.

Further more because there exist a distance function $d_{j}(A, B)$, that is $d_{j}(A, B)=0 \Leftrightarrow A=B$.

Then we have $d(A, B)=0 \Leftrightarrow A=B$

Hence, to any $(A, B) \in \operatorname{VIFS}(X)$,

$d(A, B)=f\left(d_{1}(A, B), d_{2}(A, B), \ldots, d_{s}(A, B)\right)$

is distance function.

Definition 3.2. Let $d(A, B)=W\left(\theta_{1}, \theta_{2}, \ldots, \theta_{n}\right)$, where

1. To any $(A, B) \in \operatorname{VIFS}(X)$ we have

$\theta_{i}=\left\{E_{i}^{-}\left[\phi_{i}^{-}\left(\left|\mu_{A}{ }^{-}-\mu_{B}{ }^{-}\right|\right), \varphi_{i}{ }^{-}\left(\left|v_{A}{ }^{-}-v_{B}{ }^{-}\right|\right)\right)\right.$,

$\left.E_{i}^{+}\left[\phi_{i}^{+}\left(\mu_{A}^{+}-\mu_{B}^{+} \mid\right), \varphi_{i}^{+}\left(\left|v_{A}^{+}-v_{B}^{+}\right|\right)\right\}\right\}$

2. $W:[0,1]^{n} \rightarrow[0,1]$ with properties:

1) $x_{1}=x_{2}=\ldots=x_{n}=0 \Leftrightarrow W\left(x_{1}, x_{2}, \ldots, x_{n}\right)=0$

2) To any $x_{i}(i=1,2, \ldots, s), W$ is a decreasing function.

3. $\theta_{i}:[0,1] \times[0,1] \rightarrow[0,1](i=1,2, \ldots, n)$ with properties:

1) $\theta_{i}(x, y)=0 \Leftrightarrow x=y=0$

2) $\theta_{i}$ is a increasing function to any variable.

4. $E_{i}{ }^{-}, E_{i}^{+}:[0,1] \times[0,1] \rightarrow[0,1](i=1,2, \ldots, n)$ with properties:

1) $E_{i}^{-}(x, y)=0 \Leftrightarrow x=y=0 \Leftrightarrow E_{i}^{+}(x, y)=0$

2) $E_{i}^{-}, E_{i}^{+}$is a increasing function to any variable.

5. $\phi_{i}^{-}, \phi_{i}^{+}, \varphi_{i}^{-}, \varphi_{i}^{+}:[0,1] \rightarrow[0,1](i=1,2, \ldots, n)$

with properties:

1) $\phi_{i}^{-}(x)=0 \Leftrightarrow \phi_{i}^{+}(x)=0 \Leftrightarrow x=0$

$$
\Leftrightarrow \varphi_{i}^{-}(x)=0 \Leftrightarrow \varphi_{i}^{+}(x)=0
$$

2) $\phi_{i}^{-}, \phi_{i}^{+}, \varphi_{i}^{-}, \varphi_{i}^{+}$are increasing functions. 
Theorem 3.3. $d(A, B)$ in definition 3.2 is a distance function.

Prove: 1) let $(A, B) \in \operatorname{VIFS}(X)$, if $A=B$, then

$$
\begin{aligned}
& \mu_{A}{ }^{-}-\mu_{B}{ }^{-}=0, v_{A}{ }^{-}-v_{B}{ }^{-}=0, \\
& \mu_{A}{ }^{+}-\mu_{B}{ }^{+}=0, v_{A}{ }^{+}-v_{B}{ }^{+}=0 .
\end{aligned}
$$

From properties 2) of $W, \theta_{i}, E_{i}^{-}, E_{i}^{+}, \phi_{i}^{-}, \phi_{i}^{+}, \varphi_{i}^{-}, \varphi_{i}^{-}$we know

$$
A=B \Leftrightarrow d(A, B)=0
$$

2) from definition 3.2 , it is easy to prove that

$$
d(A, B)=d(B, A) .
$$

3) let $A \leq B \leq C,(A, B) \in \operatorname{VIFS}(X)$

$$
\begin{aligned}
& v_{A}{ }^{-}-v_{C}{ }^{-} \geq v_{A}{ }^{-}-v_{B}{ }^{-} \geq 0 \geq \mu_{A}{ }^{-}-\mu_{B}{ }^{-} \geq \mu_{A}{ }^{-}-\mu_{C}{ }^{-} \\
& v_{A}{ }^{+}-v_{C}{ }^{+} \geq v_{A}{ }^{+}-v_{B}{ }^{+} \geq 0 \geq \mu_{A}{ }^{+}-\mu_{B}{ }^{+} \geq \mu_{A}{ }^{+}-\mu_{C}{ }^{+} \text {Hence } \\
& \\
&\left|v_{A}{ }^{-}{ }^{-} v_{C}{ }^{-}\right| \geq\left|v_{A}{ }^{-}-v_{B}{ }^{-}\right| \\
&\left|\mu_{A}{ }^{-}{ }^{-} \mu_{C}{ }^{-}\right| \geq\left|\mu_{A}{ }^{-}-\mu_{B}{ }^{-}\right| \\
& \\
&\left|v_{A}{ }^{+}{ }^{-} v_{C}{ }^{+}\right| \geq\left|v_{A}{ }^{+}-v_{B}{ }^{+}\right| \\
&\left|\mu_{A}{ }^{+}-\mu_{C}{ }^{+}\right| \geq\left|\mu_{A}{ }^{+}-\mu_{B}{ }^{+}\right|
\end{aligned}
$$

From definition 3.2 we know $\phi_{i}^{-}, \phi_{i}{ }^{+}, \varphi_{i}^{-}, \varphi_{i}{ }^{+}$all satisfy their properties 2), hence

$$
\begin{aligned}
\phi_{i}^{+} \mid v_{A}{ }^{+}-v_{C}+ & \geq \phi_{i}^{+}\left|v_{A}^{+}-v_{B}^{+}\right| \\
\varphi_{i}^{+}\left|\mu_{A}{ }^{+}-\mu_{B}^{+}\right| & \geq \varphi_{i}^{+}\left|\mu_{A}^{+}-\mu_{C}^{+}\right| \\
\phi_{i}{ }^{+}\left|v_{A}{ }^{-}-v_{C}{ }^{-}\right| & \geq \phi_{i}^{-}\left|v_{A}{ }^{-}-v_{B}{ }^{-}\right| \\
\varphi_{i}{ }^{-}\left|\mu_{A}{ }^{-}-\mu_{B}{ }^{-}\right| & \geq \varphi_{i}{ }^{-}\left|\mu_{A}{ }^{-}-\mu_{C}{ }^{-}\right|
\end{aligned}
$$

And in definition 3.2, $E_{i}{ }^{-}, E_{i}^{+}$all satisfy their properties 2), hence

$$
\begin{gathered}
E_{i}{ }^{-}\left[\phi_{i}{ }^{-}\left(\mu_{A}{ }^{-}-\mu_{B}{ }^{-}\right), \phi_{i}{ }^{-}\left(v_{A}{ }^{-}-v_{B}{ }^{-}\right)\right] \leq E_{i}{ }^{-}\left[\varphi_{i}{ }^{-}\left(\mu_{A}{ }^{-}-\mu_{C}{ }^{-}\right), \varphi_{i}{ }^{-}\left(v_{A}{ }^{-}-v_{C}{ }^{-}\right)\right] \\
E_{i}^{+}\left[\phi_{i}^{+}\left(\mu_{A}{ }^{+}-\mu_{B}{ }^{+}\right), \phi_{i}{ }^{+}\left({v_{A}}^{+}-v_{B}{ }^{+}\right)\right] \leq E_{i}^{+}\left[\varphi_{i}^{+}\left(\mu_{A}{ }^{+}-\mu_{C}{ }^{+}\right), \varphi_{i}^{+}\left(v_{A}{ }^{+}-v_{C}{ }^{+}\right)\right]
\end{gathered}
$$

From properties 2) of $W$ we have

$$
d(A, B) \leq d(A, C)
$$

Hence, $d(A, B)$ is a distance function.

E.g. 3.1. let

1. $W=\frac{1}{n} \sum_{i=1}^{n} x_{i}$

2. $\theta_{i}(x, y)=\frac{x+y}{2}$

3. $E_{i}^{+}(x, y)=\max (x, y)=E_{i}^{+}(x, y)$

4. $\phi_{i}^{-}(x)=\phi_{i}^{+}(x)=\varphi_{i}^{-}(x)=\varphi_{i}^{+}(x)=x$

Then $d(A, B)=\frac{1}{2 n} \sum_{i=1}^{n}[\max (H)+\max (K)]$

Where

$$
\begin{gathered}
H=\left|\mu_{A}{ }^{-}\left(x_{i}\right)-\mu_{B}{ }^{-}\left(x_{i}\right)\right|,\left|v_{A}{ }^{-}\left(x_{i}\right)-v_{B}{ }^{-}\left(x_{i}\right)\right| \\
K=\left|\mu_{A}{ }^{+}\left(x_{i}\right)-\mu_{B}{ }^{+}\left(x_{i}\right)\right|,\left|v_{A}{ }^{+}\left(x_{i}\right)-{v_{B}}^{+}\left(x_{i}\right)\right|
\end{gathered}
$$


Let

$$
\mu_{A}{ }^{-}\left(x_{i}\right)=\mu_{A}{ }^{+}\left(x_{i}\right)=\mu_{A}\left(x_{i}\right) v_{A}{ }^{-}\left(x_{i}\right)=v_{A}{ }^{+}\left(x_{i}\right)=v_{A}\left(x_{i}\right) \quad i \in\{1,2, \ldots, n\}
$$

Then we have

$$
d(A, B)=\frac{1}{n} \sum_{i=1}^{n}\left[\max \left(\left|\mu_{A}\left(x_{i}\right)-\mu_{B}\left(x_{i}\right)\right|,\left|v_{A}\left(x_{i}\right)-v_{B}\left(x_{i}\right)\right|\right)\right]
$$

The similarity measure based on this distance is:

$$
s(A, B)=1-\frac{1}{n} \sum_{i=1}^{n}\left[\max \left(\left|\mu_{A}\left(x_{i}\right)-\mu_{B}\left(x_{i}\right)\right|,\left|v_{A}\left(x_{i}\right)-v_{B}\left(x_{i}\right)\right|\right)\right]
$$

This is the similarity proposed by Hung et al..

E.g. 3.2. let

$$
\begin{aligned}
& \text { 1. } W=\left[\frac{1}{n} \sum_{i=1}^{n} x_{i}\right]^{\frac{1}{p}} \\
& \text { 2. } \theta_{i}(x, y)=\left(\frac{x+y}{2}\right)^{p} \\
& \text { 3. } E_{i}^{+}(x, y)=\frac{x+y}{2}=E_{i}^{+}(x, y) \\
& \text { 4. } \phi_{i}^{-}(x)=\phi_{i}^{+}(x)=\varphi_{i}^{-}(x)=\varphi_{i}^{+}(x)=x
\end{aligned}
$$

Then

$$
\begin{gathered}
d(A, B)=\frac{1}{\sqrt[p]{n}}\left\{\sum_{i=1}^{n} \frac{1}{2^{p}}[M+N]^{p}\right\}^{\frac{1}{p}} M=\frac{\left|\mu_{A}{ }^{-}\left(x_{i}\right)-\mu_{B}{ }^{-}\left(x_{i}\right)\right|+\left|v_{A}{ }^{-}\left(x_{i}\right)-v_{B}{ }^{-}\left(x_{i}\right)\right|}{2} \\
N=\frac{\left|\mu_{A}{ }^{+}\left(x_{i}\right)-\mu_{B}{ }^{+}\left(x_{i}\right)\right|+\left|v_{A}{ }^{+}\left(x_{i}\right)-{v_{B}}^{+}\left(x_{i}\right)\right|}{2}
\end{gathered}
$$

Let

$\mu_{A}{ }^{-}\left(x_{i}\right)=\mu_{A}{ }^{+}\left(x_{i}\right)=\mu_{A}\left(x_{i}\right), v_{A}{ }^{-}\left(x_{i}\right)=v_{A}{ }^{+}\left(x_{i}\right)=v_{A}\left(x_{i}\right) \quad i \in\{1,2, \ldots, n\}$,

Then we have

$$
d(A, B)=\frac{1}{\sqrt[p]{n}}\left\{\sum_{i=1}^{n}\left[\frac{\left|\mu_{A}\left(x_{i}\right)-\mu_{B}\left(x_{i}\right)\right|+\left|v_{A}\left(x_{i}\right)-v_{B}\left(x_{i}\right)\right|}{2}\right]^{p}\right\}^{\frac{1}{p}}
$$

The similarity measure based on this distance is:

$$
s(A, B)=1-\frac{1}{\sqrt[p]{n}}\left\{\sum_{i=1}^{n}\left[\frac{\left|\mu_{A}\left(x_{i}\right)-\mu_{B}\left(x_{i}\right)\right|+\left|v_{A}\left(x_{i}\right)-v_{B}\left(x_{i}\right)\right|}{2}\right]^{p}\right\}^{\frac{1}{p}} \text { This is the similarity proposed by }
$$

Liang et al.

\section{Summary}

In this paper, we proposed a new measure of similarity based on distance on VIFSs. These measures are useful on all existed fuzzy sets. This paper gives examples to show that these corresponding similarity measures based on our distance can involve almost all existing similarity measures. These measures are applicable on existed fuzzy sets. Thus we will focus on constructing a fuzzy query processing strategy for large scale RFS networks, which is important in our monitoring. 


\section{Acknowledgements}

This project is funded by the Qingdao Scientific and Technological development plan under Grant No. 10-3-4-1-4-jch and by the National Natural Science Foundation of China under Grant No. 60970130.

\section{References}

[1] Zheng Yang, Mo Li, Yunhao Liu, Sea Depth Measurement with Restricted Floating Sensors. The 28th IEEE Real-Time Systems Symposium, Tucson,2007, p.469-478.

[2] X.D. Liu, S.H. Zheng, and F.L. Xiong, Entropy and Subsethood for General Interval-Valued Intuitionistic Fuzzy Sets, FSKD 2005, Berlin Heidelberg, 2005, p.42-52.

[3] Shili Xiang, HockBeng Lim, KianLee Tan, Yongluan Zhou SimilarityAware Query Allocation in Sensor Networks with Multiple Base Stations. The 4th International Workshop on Data Management for Sensor Networks (DMSN'07), Austria, 2007,p.1-6.

[4] L.A. Zadeh, "Fuzzy sets", Inform. and Control, 1965, p. 338-353.

[5] K.T. Atanassov, Intuitionistic fuzzy sets. Fuzzy Sets and Systems, Elsevier, Amsterdam, 1986: p.87-97.

[6] K.T. Atanassov, G. Gargov, Interval-valued intuitionistic fuzzy sets, Fuzzy Sets and Systems, Elsevier, Amsterdam, 1989, p.343 - 349

[7] W. Xue, Q. Luo, L. Chen, and Y. Liu, "Contour map matching for event detection in sensor networks", SIGMOD, ACM, New York, 2006, p.145-156. 